\title{
Immunohistochemical detection of phospho-Akt, phospho-BAD, HER2 and oestrogen receptors $\alpha$ and $\beta$ in Malaysian breast cancer patients
}

\begin{abstract}
Activation of Akt signaling pathway has been documented in various human malignancies, including breast carcinoma. The objective of this study is to determine the incidence of Akt phosphorylation in breast tumours and its relationship with expression of ER-Ŭ, ER-6, HER2, Ki-67 and phosphorylated Bcl-2 associated death domain (p-BAD). Immunohistochemical staining was performed to detect these molecules on 43 paraffin-embedded breast tumour tissues with commercially available antibodies. Eighteen (41.9\%), 3 (7.0\%), 23 (53.5\%), 35 (81.4\%), 21 (48.8\%), $29(67.4 \%)$, and $34(81.0 \%)$ of breast tumours were positive for nuclear ER-Ŭ, nuclear ER-6, membranous HER2, cytonuclear p-Akt (Thr308), p-Akt (Ser473), pBAD and Ki-67, respectively. ER-Ǔ expression was inversely correlated with HER2 and Ki67 ( $\mathrm{P}=0.041$ and $\mathrm{P}=0.040$, respectively). The p-Akt (Ser473) was correlated with increased level of p-BAD (Ser136) ( $\mathrm{P}=0.012)$. No relationship of Akt phosphorylation with HER2, ERǓ or ER- 6 was found. The p-Akt (Ser473) immunoreactivity was significantly higher in stage IV than in stage I or II ( $\mathrm{P}=0.036$ or $\mathrm{P}=0.009)$. The higher Ki-67 and lower ER-Ǔ expression showed an association with patient age of $<50$ years $(\mathrm{P}=0.004)$ and with positive nodal status $(\mathrm{P}=0.033)$, respectively. Our data suggest that the Aktphosphorylation and inactivation of its downstream target, BAD may play a role in survival of breast cancer cell. This study does not support the simple model of linear HER2/ PI3K/Akt pathway in breast cancer.
\end{abstract}

Keyword: Akt; Breast cancer; HER2; Oestrogen receptor 